\title{
Harnessing intelligent technologies to curb COVID-19 pandemic: taxonomy and open challenges
}

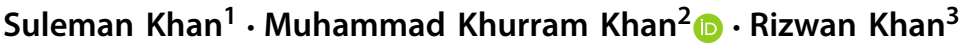

Received: 29 April 2021 / Accepted: 6 July 2021 / Published online: 30 July 2021

(c) The Author(s), under exclusive licence to Springer-Verlag GmbH Austria, part of Springer Nature 2021

\begin{abstract}
The world has changed dramatically since the outbreak of COVID-19 pandemic. This has not only affected the humanity, but has also badly damaged the world's socioeconomic system. Currently, people are looking for a magical solution to overcome this pandemic. Similarly, scientists across the globe are working to find remedies to overcome this challenge. The role of technologies is not far behind in this situation, which attracts many sectors from government agencies to medical practitioners, and market analysts. This is quite true that in a few months of time, scientists, researchers, and industrialists have come up with some acceptable innovative solutions and harnessing existing technologies to stop the spread of COVID-19. Therefore, it is pertinent to highlight the role of intelligent technologies, which play a pivotal role in curbing this pandemic. In this paper, we devise a taxonomy related to the technologies being used in the current pandemic. We show that the most prominent technologies are artificial intelligence, machine learning, cloud computing, big data analytics, and blockchain. Moreover, we highlight some key open challenges, which technologists might face to control this outbreak. Finally, we conclude that to impede this pandemic, a collective effort is required from different professionals in support of using existing and new technologies. Finally, we conclude that to stop this pandemic, machine learning approaches with integration of cloud computing using high performance computing could provision the pandemic with minimum cost and time.
\end{abstract}

Keywords COVID-19 · Technology · Artificial Intelligence · Big Data analytics · Blockchain

Mathematics Subject Classification 97P80 - 68T01 · 97Pxx

\section{Introduction}

The world has been shattered by COVID-19 pandemic [1], which was first detected in 2019 in Wuhan, China [2]. Nobody was expecting such a fast spread of this virus across

Extended author information available on the last page of the article 
the globe, which has infected and killed millions of human beings with a devastating impact on the global socio-economic system [3]. This has happened due to the frequent travellers, which resulted in the wide-spread transmission of Corona virus globally and consequently, it became a horrified pandemic in the world [4]. China has put some strong strategic planning, which helped them to control this virus after having more than 3000 deaths [5]. However, countries like India, Brazil, Italy, Spain, UK and the USA have become big victim of this pandemic so far and death rate is increasing exponentially [6].

A large number of mortalities around the world has alerted decision makers, leaders, and professionals from different areas to think about the solutions to control the spread of this virus [7]. The medical scientists started to conduct experimentations to develop vaccines while exploring symptoms of the disease [8]. Similarly, analysts based on medical advices started predicting based on the data that how this pandemic will move forward and they came up with some solution e.g. quarantine, isolation, and social distancing that could minimize spreading the virus [9]. All this requires some technologies, which can quickly be helpful for professionals to come up with some innovative solutions and ideas. The general diagram of a healthcare system and some technologies are illustrated in figure 1.

Based on our study and available data, we found that three computing technologies have become dominant in fighting this pandemic including, artificial intelligence [10], big data analytics [11], and blockchain [12]. Scientists need to investigate patients by looking towards symptoms that could include, flu, cough, and difficulties in the breath [13]. This could be managed through sensors [14], smart phones [15] devices or cameras [16], autonomous vehicles [17], and various others. Moreover, patients who are severely infected through COVID-19 are examined by checking their chest X-rays and CT scan images [18-21]. This is done by using deep learning techniques to quickly get the diagnosis results [22-26]. The problem that arises with these experiments is the availability of large datasets [27]. The data is dispersed at different locations and would be difficult to organize in such a situation where thousands of people are placed on ventilators and struggling for their life.

On the other side of the situation, scientists require high-performance computing platforms to find the genomes of this virus by finding different patterns [28]. Similarly, big data analytics provides valuable insights to the data within a short period of time, which is in great demand at this situation [29]. The businesses that require identity verifications during their daily business transactions shifted towards blockchain architecture to identify the users through smart contracts. Moreover, the data can be securely stored in distributed locations without exploiting the data integrity. This could be very useful in this current situation where healthcare data needs to be stored at a different location and processed for different reasons.

In this paper, we have provided comprehensive information related to the technologies used in this pandemic in different perspectives to give insights about its implications. Up to the best of our knowledge, this work presents first-ever taxonomy about the technologies used in the COVID-19 pandemic. Moreover, we have come up with open challenges that could be useful for researchers to work on it to come up with some quick solutions that could benefit people in this pandemic. The contribution of this work has been highlighted below: 


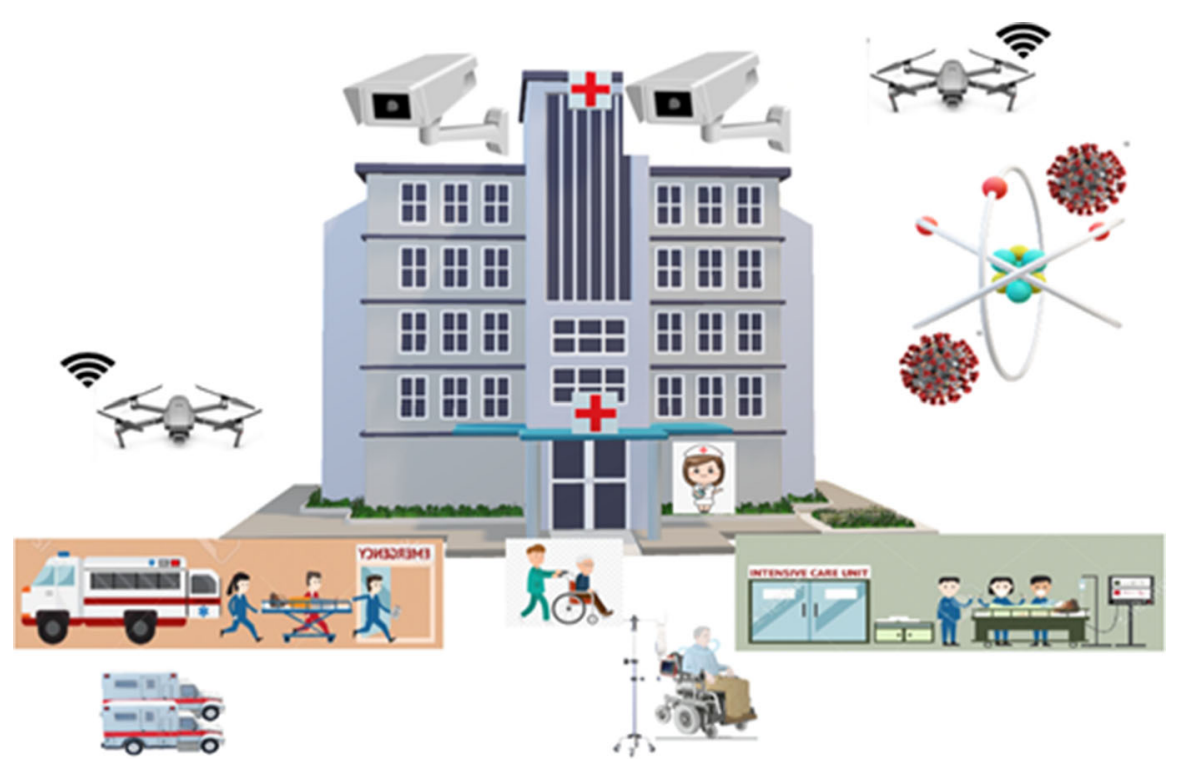

Fig. 1 Role of digital technologies in the COVID-19 pandemic

1. Devise a comprehensive taxonomy for COVID-19 with technology perspective.

2. Explained in detail about the emerging technologies used in the pandemic.

3. Provide brief discussion about open challenges faced by the technologies during the pandemic.

4. Lastly, provide recommendations based on the lesson learned.

The rest of the paper is organized as follows: Sect. 2 presents the taxonomy in terms of technology used in the pandemic. Section 3, explains the open challenges, Sect. 4 provides recommendation based on lesson learned and finally, we conclude our paper in Sect. 5.

\section{Taxonomy: technology perspective}

In this section, we provide in-depth information about the technology perspective used in COVID-19 pandemic and devise COVID-19 technology taxonomy as illustrated in Fig. 2. The taxonomy consists of five main categories including (1) Emerging and Enabling Technologies, (2) Detection \& Applications, (3) Diagnosis, (4) Traceability, and (5) Requirements. These categories provide a clear understanding of the technologies used in efforts to fight against COVID-19 pandemic.

\subsection{Emerging and enabling technologies}

This section presents the key areas of emerging and enabling technologies used in the fight against COVID-19 pandemic. We have focused on key technologies including artificial Intelligence, big data analytics, and blockchain, respectively. 


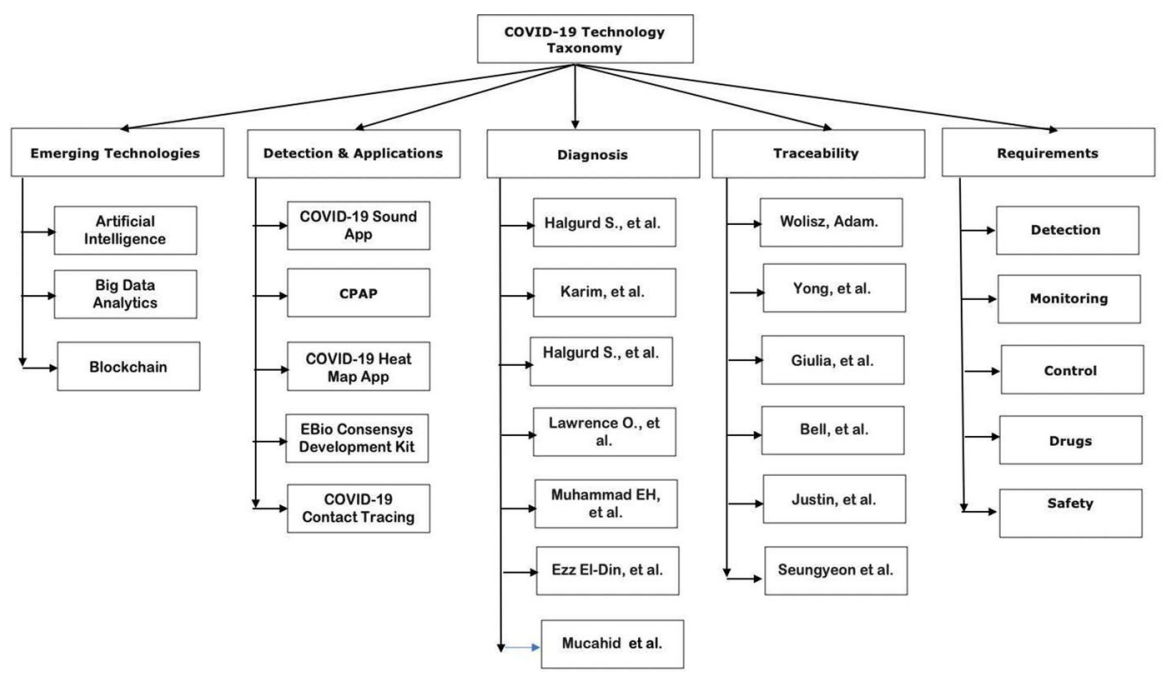

Fig. 2 Taxonomy $\rightarrow$ Technology Perspective in COVID-19 Pandemic

\subsubsection{Artificial intelligence}

Artificial Intelligence (AI) plays a key role in almost every aspect of our life. Currently, the world is suffering with COVID-19 pandemic which has shattered the socio-economic system of the world [30]. AI can support this pandemic by predicting and understanding the healthcare conditions, model risk association, and recommend solutions based on different forecasts [31-33]. The vast scope of AI supports different medical doctors and practitioners in addition to their medical expertise [34]. This could be real-time alerts and warnings or with different predictions about a patient's situation at different stages of its infection. Deep learning remains a high priority choice for different researchers in diagnosing patients through chest X-rays and CT scan images [25,35,36]. The accuracy in diagnosing COVID-19 patients could be improved with having patient's data, which is currently a great challenge to the entire world of researchers. The AI attracts researchers in treatment of COVID-19, which could be a new platform of research in this pandemic [37]. In addition, various AI techniques are used to collect the data from users to investigate user contacts and their mobility which could be vital in controlling the COVID-19. Generally, the problem AI faces in this pandemic is twofold. Firstly, the data of COVID-19 patient is not enough to get highly accurate results through AI techniques [38]. Secondly, the dispersion of the data makes things more disordered that may cause scientists to think differently.

\subsubsection{Big data analytics}

The importance of big data has increased in the last few months due to the pandemic situation of COVID-19. Medical teams across the globe are expecting from scientists to help them out with new innovative solutions by analysing the data. Currently, the data is available in the form of demographics and locations of people. There 
are numerous platforms available where people share their information voluntarily based on their situation and circumstances. This could help out big data analytics to analyse the data and extract more insights that could help different communities in the society. This could be government strategic planners, medical doctors, individuals, police and law enforcement agencies, and various others. However, the problem faced by the analytics is the correctness, timeliness, and completeness of the data. The incorrect, delivered late, or incomplete data could transform the predictions and results in incorrect outcomes. Currently, the main source of data collection is through social networks where mostly users post their location and demographics. This information is helpful in the digital world but it could violate personal privacy of users, which is a grand challenge at the moment. To convince users to provide correct information requires public confidence that can be achieved by informing them at the right time for the reasons that why their data is required, how it would be collected, where it would be stored, what information will be extracted, and how privacy will be protected. To gain this public confidence, government departments could play their role by informing the people about the importance of data communication. This could help data scientists to work on the data more accurately and come up with solutions that could help in controlling the spread of virus.

\subsubsection{Blockchain Technology}

The current times will be remembered not only due to the COVID-19 but also for the emergence of innovation of technologies and exponential digital transformation. Many companies which have been affected because of COVID-19 pandemic start thinking to use technical capabilities and creativity to operate their businesses [39]. The world economy will restart with a new twist of technology advancement after fighting successfully with this pandemic. Blockchain has become one of the important paradigms currently for companies and organizations to cope up their situation with digital transactions and identities. Many companies have started bringing their best efforts forward and come up with ice-breaking ideas against the fight of current pandemic by using decentralization and blockchain [40].

However, blockchain is not the only solution when we deal with the data that is not trusted and weak in nature as it does not need to be immutable. Beside this, blockchain has brought a lot of interest to companies in different solutions through loans origination, digital identities, tracking and tracing, payments, and providing supply chains [41]. Digital authentication [42] could help different government offices to identify the credentials and documents through using blockchain technology as there are lockdowns in almost every country so that no one can physically go to the offices. The identities are managed by the blockchain technology. Distributed ledger technology could help to store healthcare information which can be distributed securely without exploiting its data integrity [43]. This could help medical staff and government bodies to monitor the situation of this pandemic and conditions of the patients. 


\subsection{Detection systems and applications}

This section describes the key solutions that have been developed for detecting COVID19 virus with different perspectives that are highlighted in Table 1.

\subsubsection{COVID-19 sound app}

The team in University of Cambridge has developed an app called COVID-19 Sounds app which detects COVID-19 patients by checking the sound of their voice, coughing and breathing [44]. The sound is collected by the app and it uses machine learning algorithms to check that the person is suffering from COVID-19 or not. Moreover, this app also collects some basic demographic and medical information while using microphone of the smartphone. This will support data analytics with some more information parallel with the breathing and cough data. This data could be used for some more in-depth analysis used by different scientist to find accurate remedies and solutions. According to the developers of this app, they have spoken to the doctors that the breathing of the COVID-19 patient is a key feature in detecting the infected patient as it is not performed normally.

\subsubsection{Continuous positive airway pressure (CPAP)}

The Continuous Positive Airway Pressure (CPAP) is a device, which helps COVID19 patients to breathe more easily if they have lung infections and oxygen masks are not available. This new breathing aid device is developed by engineers at University College London (UCL) and Mercedes F1, which is now freely available for clinicians at University College London Hospital [45]. The device plans to help many lives and ensures that the ventilators are available for the severe ill patients. The CPAP was developed in less than 100 hours by re-engineering its previous model. The output shows that CPAP uses $70 \%$ less oxygen as compared to its previous model.

\subsubsection{Heat map app}

The heat map app is developed by a UK-based company named Evergreen Life with the help of data and health scientists [46]. This app is developed to know the current situation of the COVID-19 virus in the UK. The people are encouraged to voluntarily key-in their data, which is further shared with different leading universities in the country to analyse data. The motive behind this project is to fight against the spread of COVID-19 virus in terms of getting a clear picture of its outbreak after lockdown in the country. The statistics of the virus are provided on a daily basis. The notifications are sent to the users to perform the COVID-19 checks regularly and encourage to update their data to track out the changes in the symptoms.

\subsubsection{Shimmer3 EBio Consensys Development kit}

The ECG/Bioimpedance sensor has been developed to get real-time indications of respiration from lungs by monitoring bio impedance [47]. The sensor captures the 
electrical impulses and transfers them to Shimmers Consensys PRO software to get real-time analysis. The data can be stored on SD-card for post processing as well. The sensor helps to get information related to respiration rate, fluids in the lungs, and respiration volume. Moreover, the sensor can collect 9dof IMU data via 3-axis accelerometer, 3-axis gyroscope, and 3-axis magnetometer data. The sensor is capable enough to work for more than 2 days on data storage mode while it can last for more than 8 hours on streaming mode.

\subsubsection{Automate COVID-19 Contact tracking}

The system that is in process of development by the MIT research team and experts from other different institutions is to trace the contacts of the individuals to whom a COVID-19 infected person met based on using Bluetooth signals of its smartphone [48]. Each smartphone keepsa record of other nearby smartphones through chirps, which are random strings. The working process for this COVID-19 contact tracking is simple. Ifanyone's test is diagnosed as positive, then he needs to upload his information (chirps) to the database for last the 14 days. The other people can scan the database for their own chirps to check any match with the chirps in the database. Any match will declare that the person was in contact with the COVID-19 patient and he needs to follow instructions of the medical team. The Lincoln lab engineers have built the prototype of the system and have achieved the hardest part which was interoperability between iPhone and Android devices. In addition, Apple and Google with joint effort will produce a comprehensive solution for contact tracing, which will use operating system-level technology and application programming interfaces (APIs) [49]. The underlying technology would be using Bluetooth to reduce the spread of the virus while keeping privacy and security as its central design mechanism.

\subsection{COVID-19 diagnosis}

This section explains how COVID-19 has been diagnosed through using different technologies. We discuss current state-of-the-art literature in this regard and the literature mostly consists of artificial intelligence techniques and more specifically deep learning mechanisms to diagnose COVID-19 patients through investigating chest X-rays and CT scan images.

Halgurd et al. have proposed deep learning-based technique to detect COVID-19 from $\mathrm{X}$-rays and CT scans [50]. The X-ray and CT images are taken from multiple sources available publicly. The convolution neural network $(\mathrm{CNN})$ and modified pre-trained AlexNet Model is used for available X-ray and CT scan dataset. The drawback of the work is that the technique is used on the existing dataset due to lack of publicly available dataset of the COVID-19 patients.

This work uses deep learning techniques to automatically analyse chest X-ray images [51]. The publicly open datasets of chest X-rays have been used for training the data and using deep learning architecture to provide precision tools for diagnosing COVID-19 confirmed patients. The main focus of this work is on pneumonia infection detection as normally it has high probability that it could result in a positive COVID-19. Some 


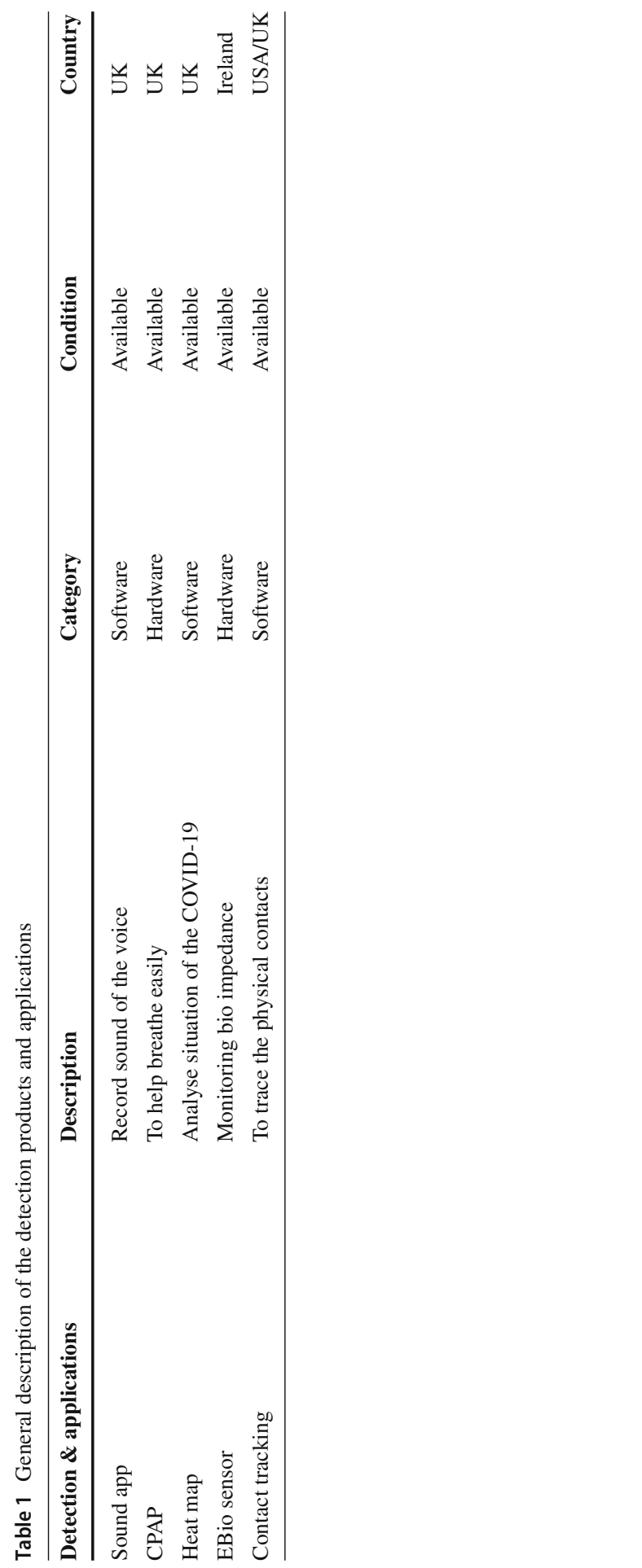


health indicators are proposed to estimate the infection and predict the condition of the patients. The results show that chest X-ray images of COVID-19 patients were successfully diagnosed with a good accuracy rate.

The authors in this work have come up with a new idea to diagnose the COVID-19 patient by using its built-in smartphone sensors [52]. To install new sensors or using different software development kits are more time consuming and expensive. Today's smartphones are powerful enough to observe different behaviour of the human through already installed sensors such as temperature, inertial, proximity, colour, humidity and other sensors. These built-in sensors should be utilized to find breathing, cough, sound, and fatigue of the human which could help medical staff to diagnose the symptoms of COVID-19 virus. The collected information from sensors is passed to artificial intelligence enabling the framework to predict the severity of the disease.

A deep learning model has been used on a small dataset containing chest X-ray images of COVID-19, viral, and bacterial patients [53]. This could help out in finding how much false positive rate is and how it could help medical practitioners in this current situation of pandemic. The dataset contains 122 COVID-19 patients' records and 4000 viral and bacterial patients. The 10-fold cross validation was used in pre-training deep convolutional neural networks on 102 patients each for COVID-19 and pneumonia cases. All cases were successfully classified for COVID-19 patients however; the only constraint is the availability of the small dataset for the experiment.

Chowdhury et al. have used pre-processing and deep learning techniques to detect COVID-19 patients by analysing the digital X-rays [54]. The public dataset has been used to check the accuracy to diagnose patients with the symptoms of COVID-19 virus. In the experiment, 190 COVID-19, 1341 normal, and 1345 viral pneumonia chest Xrays have been used. Four different deep Convolutional Neural Networks (CNN) were used to train and validate 2500 combined images. The authors have received upto $100 \%$ accuracy for combination of its image schemes such as (normal-COVID-19pneumonia) and (normal, viral and COVID-19 pneumonia).

EED Hemdan et al. have introduced a new deep learning technique with a name called COVIDX-Net [55]. This technique helps radiologists to diagnose COVID-19 patients automatically to save their time. The COVIDX-Net work on seven different deep convolutional neural network models including modified Visual Geometry Group Network (VGG19) and Google MobileNet. The experiment was run on a short dataset, including chest X-rays of 50 with 25 confirmed cases of COVID-19. The authors suggested that the performance of MobileNetV2 could be improved through using some smart devices due to its high processing power.

Barstugan et al have used machine learning methods to detect COVID-19 patients by analysing CT scan images [56]. Four different datasets were used with patch size $16 \times 16,32 \times 32$, 48x48, 64x64 from 150 images. Further, the classification performance was enhanced by applying the feature extraction process to the patches. Five different feature extraction mechanisms were used to extract different features while support vector machine classifier used to classify them. The $99.68 \%$ accuracy was achieved with the GLSZM feature extraction method for 10-fold cross-validation. 


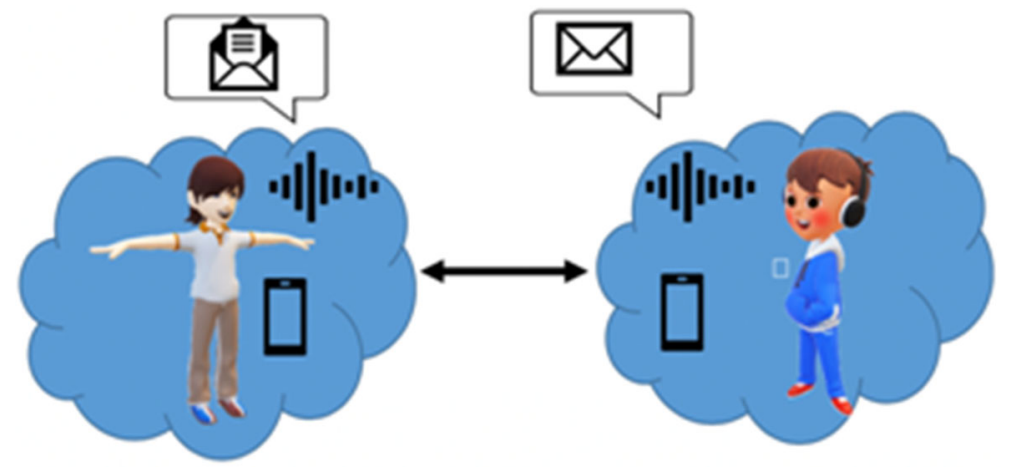

Fig. 3 Social distancing $\rightarrow$ COVID-19

\subsection{COVID-19 traceability}

This section describes how COVID-19 infected patients have been traced by using technologies. In the current literature, different researchers have used different mechanisms to trace infected persons and their contacts in COVID-19 pandemic. We have explained in detail the state-of-the-art literature, which performs traceability in finding affected people.

Adam Woliszhas presented a mechanism to back track the contacts of the affected COVID-19 patient so that it could not spread the virus further in the locality [57]. This approach is claimed to keep data privacy of the users without violating the data laws. The technique uses a smartphone which is assigned with a Pseudo-ID called PID. The PID is some sequence of symbols especially used to track the spread of the disease. The smartphone is enabled with Bluetooth and has a running contact identification service. The smartphone should have the ability to identify other Bluetooth devices, check its distance either it is close or far enough, and should exchange information with it. The log of its smartphone would be stored in the cloud or its phone, and if the patient is diagnosed with COVID-19 virus the medical doctors would generate a certificate of infection by providing its PID-ID only. The notification service running on the patient smartphone would notify all his contacts for telling about its infection. In this study, Yong et al. have used time-dependent Susceptible, Exposed, Infectious, and Recovered (SEIR) model to predict time series of the COVID-19 virus in different provinces and cities of China [58]. The results have shown the infection rate and spatiotemporal variation in the recovery rate due to different strategy used by China to control the spread of this virus. The SEIR model was applied to predict the situation of the USA, Japan, Italy, and South Korea. The results were quite different due to monitoring and fighting strategies against the virus in different countries. Moreover, a stochastic model using random walk particle tracking scheme and mixing-limited bimolecular reaction model was developed to analyse non-pharmaceutical approaches in the fight of this pandemic. The results show that social distancing could be a good solution to control the spread of the virus instead of a self-quarantined approach. The general diagram of social distancing is shown in Fig. 3. 
Giordano et al have proposed a model to discriminate between COVID-19 infection patients based on the diagnosis or condition of their symptoms [59]. The diagnosed patient is not much dangerous in spreading the virus as compared to non-diagnose one as he is self-quarantine. A SIDARTHE model is proposed based on a mean-field epidemiological model to differentiate between diagnose and non-diagnose cases. This is supported with some other features including severity of illness, non-life-threatening cases, symptomatic cases, and other which might require intensive care units for their treatment. The results show that social distancing is required on an urgent basis in Italy to stop spreading this massive outbreak of COVID-19.

Contact tracing is important to track the contacts of the infected person with COVID19 virus. In this work, authors have come up with two solutions that ensure privacy preservation of the users [60]. First solution is based on a private message that is an extension of the currently implemented method known as Trace-Together while the second solution allows two parties to exchange secrets at time of interaction which is supported by homomorphic encryption. Both of these solutions provide privacy of the infected users which is sometimes not considered by the current solutions. The results also show that both of the solutions are efficient and inexpensive, which allow them to be practical especially in this current fight against COVID-19 pandemic.

Chan et al. have presented privacy sensitive protocols and different methods to support public health organization goals while using computing technologies to stop spreading COVID-19 virus [61]. The work supports security and privacy risk associated with third-party free approaches for assisting mobile contact tracing. The approach also looks at the inferential risks involved with contact tracing systems, which itself might increase the danger of de-anonymizing information to the user. The information between different users is passed out through Bluetooth and if both users within the same location are affected (it might be either positive or negative), then other users in proximity are informed by uploading information to the server. The approach also integrated Mobile-Assisted Contact Tracing Interviews and narrowcasting mechanisms to support the approach with more accurate information which could help public health organizations.

Seungyeon et al have presented a new way to perform contact tracing using smartphones [62]. The smartphone magnetometer readings are used to trace the contacts for infected people. The reading magnetometer of smartphone is highly linear correlated when two phones are close to each other. This could act as a proxy to find the close human contact events while serving to enable contact tracing automatically. The measure is performed in different situations and based on sensitivity, likelihood ratio, specificity, and odds ratios have resulted with high accuracy by finding contacts from no contacts.

\subsection{Technology requirements}

In this section, we discuss the demanding requirements of the technologies to overcome COVID-19 virus that are highlighted in Table 2. We have divided these demanding issues into five general categories which cover almost all aspects of this pandemic i.e. detection, monitoring, control, drugs, and safety. 


\subsubsection{Detection}

The most important aspect of this pandemic is to detect corona virus at its early stage [63]. The question arises how an existing technology can detect COVID-19 early before it is disseminated to the world. Artificial intelligence can help out in this regard to predict the spread of the virus at its early stage [64]. The Canadian based digital health company named BlueDot uses AI platforms to detect infectious diseases and protect humans [65]. They have also predicted the COVID-19 virus by analyzing the data retrieved from airlines data, news reports, and animal disease reports at its early stage. These data are further analysed by epidemiologists.

\subsubsection{Monitor}

It is quite important to monitor the outbreak situation of COVID-19 pandemic [66]. It requires support of the technologies to collect data from different sources and make it available to the world to know about the current situation. The Johns Hopkins University in the USA has developed an online dashboard, which shows new cases, recoveries, and confirms deaths on a daily basis by extracting the data from different sources [67]. The data is also down loadable as a Google sheet for further analysis.

\subsubsection{Control}

The most important phase of this COVID-19 outbreak is to introduce technologies, which can help out to control corona virus patients. The earlier control is important to overcome the burden on the hospitals. It could result in reserving ventilators, oxygen, medical equipments, and medical staff for the severe infected patients. Different companies are working on the development of kits, including sensors to diagnose COVID-19 patients through different symptoms to get early treatment before the situation becomes worse. This may require to capture breath, cough, or other actions to sense the condition of the humans.

\subsubsection{Drugs}

To come up with a new vaccine for this new virus is a great challenge for all the world. The vaccine could act like a drug to cure the illness as in this pandemic is virus which causes many symptoms. The scientists need to understand the genome sequence of the COVID-19 to come up with a vaccine. The sequence of findings genome requires high technology to fasten the process and this happened to COVID-19 genome, as Chinese scientists have sequenced the genome in less than a month while using powerful technologies. The sequencing of the genome is crucial for development of vaccines.

\subsubsection{Safety}

Different studies mentioned that virus can transfer from human to human [68]. This makes the life of medical staff at risk in the hospitals [69]. Therefore, it becomes 
Table 2 General requirements for technologies in COVID-19 pandemic

\begin{tabular}{lll}
\hline Requirements & Explanation & Technology \\
\hline Detection & Detect the spread & AI based platforms \\
Monitoring & Observe the outbreak & Online dashboards \\
Control & Diagnose the patients & Sensors, smartphones, apps \\
Drugs & New vaccines & High performance computing \\
Safety & Protect the humans & Through robots \\
\hline
\end{tabular}

cumbersome and challenging for medical doctors or practitioners to treat COVID-19 patients. In this situation, robots can play an important role to support the medical staff [70]. For instance, a robot can go to the patient on the bed and capture his images, sense body actions, and communicate to record the voice. This information would be vital for doctors to observe the condition of the patients without putting their life in danger. Many hospitals have started using robots in treating COVID-19 patients.

\section{Open challenges}

This section describes some key challenges that might be faced by the world in using technologies against the fight of COVID-19 as shown in Fig. 4.

\subsection{Hardware cost}

The solutions to diagnose COVID-19 patients use sensors to observe the conditions of the patients actions such as respiration, lungs fluid, breathing, and cough [71]. This requires patients to be connected with the sensors, which might be a challenge to do for each and every person in the locality. The availability of sensors to all the public is important to have accurate results and control on the outbreak. This could be a costly task to develop sensors in large with short span of time and make its availability to the public, especially during this pandemic situation. The high cost of the sensors wouldn't be possible to afford by the developing and vulnerable nations.

\subsection{Trustworthiness}

To control the outbreak of COVID-19 virus requires accurate real-time information to monitor the situation [67]. This demands volunteer actions from the affected patients to update their information regularly on different portals and apps provided by different companies. The question arises is that an easy job for the patients who are already depressed with tension and worried about their life within this pandemic. Some malicious users can upload fake or wrong information which can result in high false positive [72,73]. Trustworthiness is an important factor to properly monitor COVID-19 pandemic situation that has spread throughout the globe [74]. 


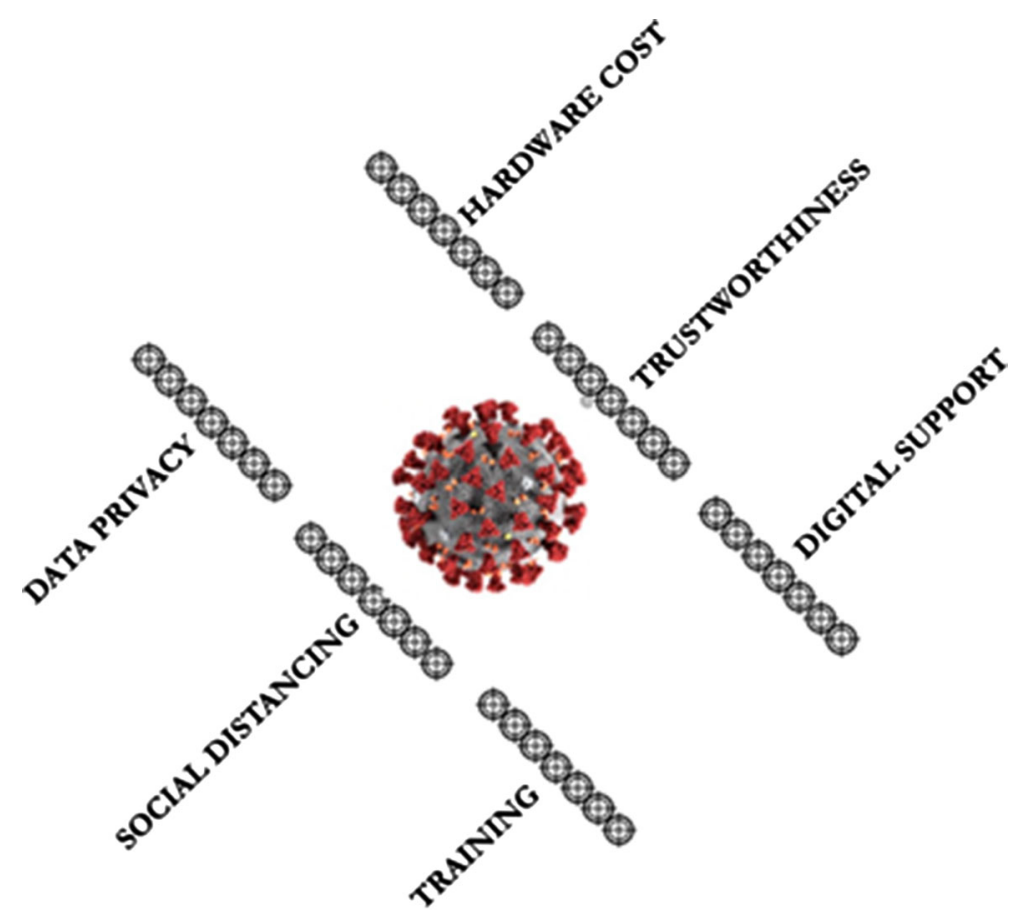

Fig. 4 Open challenges for technologies $\rightarrow$ Fight against COVID-19

\subsection{Training}

Different solutions have been introduced in the last few months to solve COVID-19 problem. One of the solutions is to use different apps and sensors, which help out to collect some useful information related to patients. Mostly, the patients are elderly people who are not familiarized with latest technologies which makes it difficult to use those sensors to operate $[75,76]$. A proper knowledge would be required to the people who could easily use these technologies that are being introduced to fight COVID-19 pandemic. It is a great challenge to quickly train the elderly people who are isolated at home with having less knowledge, technology know-how and information.

\subsection{Data privacy}

Patients need to upload their medical information to different online portals voluntarily. This might increase the risk of data privacy of the users [77]. The companies need to ensure that they have secure platforms available for the users to upload their information. Mostly, the focus of companies is on the information itself rather than data security as a solution. This could motivate malicious users to take advantages from these situations to exploit the data [78]. Any data breach could make the life of the patient in danger. 


\subsection{Digital support solution}

The big challenge faced by most of the government across the world is to convince people to stay at home to control the outbreak of COVID-19 [79,80]. Can technologies play its part to achieve this milestone? Some companies have started building online games for kids [81] to make them stay at home while some groceries retailers start building apps to deliver daily usage items to homes [82] and so on. Online social networking could be of great interest to connect different family members with each other while reducing the distances. There are great opportunities for technology vendors to come up with some novel ideas to help people to remain in their homes by obeying the government orders to overcome this massive pandemic.

\subsection{Social distance}

To reduce the spread of COVID-19 virus, it is strongly recommended to keep social distancing among the people $[9,83]$. With this important message, can technology help out people to obey social distancing rules to stop transmitting virus from human to human? Different social distancing apps could make this easy but how this could be achieved. Many suggestions are floating on the Internet that sensors in smartphones could help to sense the distances among people. However, there are still great challenges related to smartphones to find the accurate distances among people. One of the challenges include communication between heterogeneous smartphone devices such as iPhone and Android smartphones. By solving this challenge accurately could help in finding social distancing easily.

\section{Lesson learned}

This study has shown that a collective effort is required from a medical practitioner, front line workers, technologists, researchers, and industrialists to curb COVID-19 pandemic. Each personnel from different professions needs to combine and cooperate their capabilities with other departments to control the spread of this virus. However, the role of IT experts becomes more demanding to develop new technologies that could cope with this new pandemic which has been faced by the world for the first time. The solutions could be in terms of enhancing previous technologies or developing new technologies to help to spread this pandemic.

One of the key focus remain on the machine learning approaches which could help out in classifying and clustering the data while using high performance computing machines. This could be enhanced more while connecting with IoT which could sense the data from wearables, static sensors, mobile devices and various other means of sources and distributed data to the servers for further investigation. The leading IT and technology companies need to combine their efforts in providing new solutions with less time and efforts to facilitate the world in this situation. We hope that this pandemic would fade soon with the great efforts made by all stakeholders and life would return to the normal routine. 


\section{Conclusion}

Today in the digital ecosystem, devices are interconnected that contribute from data collection to data analytics in real-time. These could be devices and instruments in hospitals or clinics that provide valuable data to AI systems for understanding healthcare problems and predict different risks. The role of technologies in healthcare is vital and this is what we have discussed in this paper. The technologies have enabled different solutions in the COVID-19 pandemic which helps in monitoring, diagnosing, and controlling the virus. This could be in terms of developing patterns of pandemic genomes or it could be social distancing solutions through different applications.

All the technologies are contributing to the global cause to stop spreading the COVID19 virus. We have found out that artificial intelligence, big data analytics, and blockchain are the most dominant technologies used in this pandemic. However, some challenges still need to be addressed on an urgent basis to strengthen the capabilities of the technologies. This requires attention from researchers and industrialists all over the globe.

Acknowledgements This paper is supported by Researchers Supporting Project number (RSP-2021/12), King Saud University, Riyadh, Saudi Arabia.

Funding There is no funding source for this work.

\section{Declarations}

Conflict of interest The authors declare that they have no conflict of interest.

Ethical approval This article does not contain any studies with human participants or animals performed by any of the authors.

\section{References}

1. Organization WH (2020) Coronavirus disease 2019 (COVID-19): situation report, 72

2. Wu JT, Leung K, Bushman M, Kishore N, Niehus R, de Salazar PM, Leung GM (2020) Estimating clinical severity of COVID-19 from the transmission dynamics in Wuhan, China. Nat Med 26(4):506510

3. Shirouyehzad H, Jouzdani J, Khodadadi Karimvand M (2020) Fight against COVID-19: a global efficiency evaluation based on contagion control and medical treatment. J Appl Res Ind Eng 7(2):109120

4. Chinazzi M, Davis JT, Ajelli M, Gioannini C, Litvinova M, Merler S, Vespignani A (2020) The effect of travel restrictions on the spread of the 2019 novel coronavirus (COVID-19) outbreak. Science 368(6489):395-400

5. Zhou F, Yu T, Du R, Fan G, Liu Y, Liu Z, Cao B (2020) Clinical course and risk factors for mortality of adult inpatients with COVID-19 in Wuhan, China: a retrospective cohort study. The lancet 395(10229):1054-1062

6. Roser M, Ritchie H, Ortiz-Ospina E (2020) Coronavirus Disease (COVID-19)-Statistics and Research, Our World in Data

7. Gozes et al. O (2020) Rapid ai development cycle for the coronavirus (covid-19) pandemic: initial results for automated detection \& patient monitoring using deep learning ct image analysis, arXiv preprint arXiv:2003.05037 
8. Prompetchara E, Ketloy C, Palaga T (2020) Immune responses in COVID-19 and potential vaccines: lessons learned from SARS and MERS epidemic. Asian Pac J Allergy Immunol 38(1):1-9

9. Anderson RM, Heesterbeek H, Klinkenberg D, Hollingsworth TD (2020) How will country-based mitigation measures influence the course of the COVID-19 epidemic? The Lancet 395(10228):931934

10. Hu Z, Ge Q, Jin L, Xiong M (2020) Artificial intelligence forecasting of covid-19 in china, arXiv preprint arXiv:2002.07112

11. Wang CJ, Ng CY, Brook RH (2020) Response to COVID-19 in Taiwan: big data analytics, new technology, and proactive testing. JAMA

12. Mashamba-Thompson, Tivani P, Ellen Debra C (202) Blockchain and artificial intelligence technology for novel coronavirus disease-19 self-testing. Vol. 198

13. Lai C-C, Shih T-P, Ko W-C, Tang H-J, Hsueh P-R (2020) Severe acute respiratory syndrome coronavirus 2 (SARS-CoV-2) and corona virus disease-2019 (COVID-19): the epidemic and the challenges. Int J Antimicrob Ag 55:105924

14. Allam Z, Jones DS On the coronavirus (COVID-19) outbreak and the smart city network: universal data sharing standards coupled with artificial intelligence (AI) to benefit urban health monitoring and management, in Healthcare, 2020, vol. 8, no. 1: Multidisciplinary Digital Publishing Institute, p. 46

15. Rao ASS, Vazquez JA (2020) Identification of COVID-19 can be quicker through artificial intelligence framework using a mobile phone-based survey in the populations when cities/towns are under quarantine. Infec Control Hosp Epidemiol 41:826-830

16. Greenhalgh T, Koh GCH, Car J (2020) Covid-19: a remote assessment in primary care, Bmj, vol. 368

17. Khalid $\mathrm{M}$ et al (2021) Autonomous transportation in emergency healthcare services: framework. Challenges, and future work. IEEE Internet of Things Mag 4(1):28-33

18. Cohen JP, Morrison P, Dao L (2020) COVID-19 image data collection, arXiv preprint arXiv:2003.11597

19. Cheng S-C et al (2020) First case of Coronavirus disease 2019 (COVID-19) pneumonia in Taiwan. J Formos Med Assoc 119:747

20. Zhang J, Xie Y, Li Y, Shen C, Xia Y (2020) COVID-19 screening on chest X-ray images using deep learning based anomaly detection, arXiv preprint arXiv:2003.12338

21. Mossa-Basha M, Meltzer CC, Kim DC, Tuite MJ, Kolli KP, Tan BS (2020) Radiology Department Preparedness for COVID-19: Radiology Scientific Expert Panel, Radiology, p. 200988

22. Xu et al. X (2020) Deep learning system to screen coronavirus disease 2019 pneumonia, arXiv preprint arXiv:2002.09334

23. Shan+ et al. F (2020) Lung infection quantification of COVID-19 in CT images with deep learning, arXiv preprint arXiv:2003.04655

24. Wang et al. S (2020) A deep learning algorithm using CT images to screen for Corona virus disease (COVID-19), medRxiv

25. Wang L, Wong A (2020) COVID-Net: A tailored deep convolutional neural network design for detection of COVID-19 cases from chest radiography images, arXiv preprint arXiv:2003.09871

26. Zhavoronkov A et al (2020) Potential COVID-2019 3C-like protease inhibitors designed using generative deep learning approaches. Insilico Med Hong Kong Ltd A 307:E1

27. Loey M, Smarandache F, Khalifa NEM (2020) Within the lack of COVID-19 benchmark dataset: a novel GAN with deep transfer learning for Corona-virus detection in chest X-ray images

28. Smith M, Smith JC (2020) Repurposing therapeutics for covid-19: Supercomputer-based docking to the sars-cov-2 viral spike protein and viral spike protein-human ace2 interface

29. Zhou et al. C (2020) COVID-19: challenges to GIS with big data, Geography and Sustainability

30. Nguyen TT Artificial intelligence in the battle against Coronavirus (COVID-19): a survey and future research directions

31. McCall B (2020) COVID-19 and artificial intelligence: protecting health-care workers and curbing the spread, The Lancet Digital Health

32. Li et al. L (2020) Artificial intelligence distinguishes covid-19 from community acquired pneumonia on chest ct, Radiology, p. 200905

33. Ting DSW, Carin L, Dzau V, Wong TY (2020) Digital technology and COVID-19. Nat Med 26(4):459_ 461

34. Bandyopadhyay SK, Dutta S (2020) Machine learning approach for confirmation of COVID-19 cases positive, negative, death and release, medRxiv,

35. Huang L et al (2020) Serial quantitative chest CT assessment of COVID-19: deep-learning approach. Radiol Cardiothorac Imagin 2(2):e200075 
36. Narin A, Kaya C, Pamuk Z (2020) Automatic detection of coronavirus disease (COVID-19) using X-ray images and deep convolutional neural networks, arXiv preprint arXiv:2003.10849

37. Hu Z, Ge Q, Li S, Boerwincle E, Jin L, Xiong M (2020) Forecasting and evaluating intervention of Covid-19 in the World, arXiv preprint arXiv:2003.09800

38. Zhao J, Zhang Y, He X, Xie P (2020) COVID-CT-dataset: a CT scan dataset about COVID-19, arXiv preprint arXiv:2003.13865

39. Koonin LM (2020) Novel coronavirus disease (COVID-19) outbreak: now is the time to refresh pandemic plans. J Bus Contin Emerg Plan 13(4):1-15

40. Corbet S, Hu Y, Lucey BM, Oxley L (2020) Aye Corona! The contagion effects of being named Corona during the COVID-19 pandemic, the contagion effects of being named Corona during the COVID-19 pandemic (March 26, 2020)

41. Singhal B, Dhameja G, Panda PS (2018) Beginning blockchain: a beginner's guide to building blockchain solutions. Springer, Berlin

42. Fisher J, Sanchez MH (2016) Authentication and verification of digital data utilizing blockchain technology, ed: Google Patents

43. Kuo T-T, Kim H-E, Ohno-Machado L (2017) Blockchain distributed ledger technologies for biomedical and health care applications. J Am Med Inform Assoc 24(6):1211-1220

44. New app collects the sounds of COVID-19. https://www.cam.ac.uk/research/news/new-app-collectsthe-sounds-of-covid-19. Accessed 12 Apr 2020. (2020)

45. Designs for life-saving breathing aid to be made freely available. https://www.mercedesamgf1.com/ en/news/2020/04/ucl-uclh-f1-project-pitlane-start-delivery-breathing-aids-nhs-hospitals/ (accessed $12 / 04 / 2020$

46. COVID-19 heat map. https://www.evergreen-life.co.uk/covid-19-heat-map (accessed 12/04/2020)

47. Shimmer3 EBio Consensys Development Kit. https://www.shimmersensing.com/products/ development-kits/ (accessed 12/04/2020)

48. MIT's COVID-19 app uses smartphones. Bluetooth to anonymously spot disease contacts. https:// www.mobihealthnews.com/news/mits-covid-19-app-uses-smartphones-bluetooth-anonymouslyspot-disease-contacts (accessed 12/04/2020

49. Apple and Google partner on COVID-19 contact tracing technology. Apple. https://www.apple.com/ newsroom/2020/04/apple-and-google-partner-on-covid-19-contact-tracing-technology/ (accessed 14-04-2020

50. Maghdid HS, Asaad AT, Ghafoor KZ, Sadiq AS, Khan MK (2020) Diagnosing COVID-19 pneumonia from X-Ray and CT images using deep learning and transfer learning algorithms, arXiv preprint arXiv:2004.00038

51. Hammoudi et al. K (2020) Deep learning on chest X-ray images to detect and evaluate pneumonia cases at the era of COVID-19, arXiv preprint arXiv:2004.03399

52. Maghdid HS, Ghafoor KZ, Sadiq AS, Curran K, Rabie K (2020) A novel ai-enabled framework to diagnose coronavirus covid 19 using smartphone embedded sensors: design study, arXiv preprint arXiv:2003.07434

53. Hall LO, Paul R, Goldgof DB, Goldgof GM (2020) Finding COVID-19 from chest X-rays using deep learning on a small dataset, arXiv preprint arXiv:2004.02060

54. Chowdhury et al. ME (2020) Can AI help in screening Viral and COVID-19 pneumonia?, arXiv preprint arXiv:2003.13145

55. El-Din Hemdan E, Shouman MA, Karar ME (2020) COVIDX-Net: a framework of deep learning classifiers to diagnose COVID-19 in X-ray images, arXiv, p. arXiv: 2003.11055

56. Barstugan M, Ozkaya U, Ozturk S (2020) Coronavirus (COVID-19) Classification using CT images by machine learning methods, arXiv preprint arXiv:2003.09424

57. Wolisz A (2020) A fully distributed, privacy respecting approach for back-tracking of potentially infectious contacts, arXiv preprint arXiv:2003.14243

58. Zhang Y, Yu X, Sun H, Tick GR, Wei W, Jin B (2020) COVID-19 infection and recovery in various countries: Modeling the dynamics and evaluating the non-pharmaceutical mitigation scenarios, arXiv preprint arXiv:2003.13901

59. Giordano et al. G (2020) A SIDARTHE model of COVID-19 epidemic in Italy, arXiv preprint arXiv:2003.09861

60. James Bell DB, Hicks Chris, Crowcroft Jon (2020) TraceSecure: towards privacy preserving contact tracing, arXiv preprint: arXiv:2004.04059 
61. Chan et al. J (2020) PACT: Privacy sensitive protocols and mechanisms for mobile contact tracing, arXiv preprint arXiv:2004.03544

62. Jeong S, Kuk S, Kim H (2019) A smartphone magnetometer-based diagnostic test for automatic contact tracing in infectious disease epidemics. IEEE Access 7:20734-20747

63. Ai et al. T (2020) Correlation of chest CT and RT-PCR testing in coronavirus disease 2019 (COVID-19) in China: a report of 1014 cases, Radiology, p. 200642

64. Vaishya R, Javaid M, Khan IH, Haleem A (2020) Artificial Intelligence (AI) applications for COVID-19 pandemic. Diabetes Metab Syndr Clin Res Rev 14(4):337-339

65. How this Canadian start-up spotted coronavirus before everyone else knew about it. https://www.cnbc. com/2020/03/03/bluedot-used-artificial-intelligence-to-predict-coronavirus-spread.html (accessed $12 / 04 / 2020$

66. Burke RM (2020) Active monitoring of persons exposed to patients with confirmed COVID-19-United States, January-February. MMWR Morb Mortal Wkly Rep 69:2020

67. Dong E, Du H, Gardner L (2020) An interactive web-based dashboard to track COVID-19 in real time. Lancet Infect Dis

68. Bernheim et al. A (2020) Chest CT findings in coronavirus disease-19 (COVID-19): relationship to duration of infection, Radiology, p. 200463

69. $\mathrm{Ng} \mathrm{K}$ et al (2020) COVID-19 and the risk to health care workers: a case report. Ann Intern Med 172:766-767

70. Yang et al. G-Z (2020) Combating COVID-19-The role of robotics in managing public health and infectious diseases, ed: Science Robotics

71. Rashid MT, Wang D (2020) CovidSens: a vision on reliable social sensing based risk alerting systems for COVID-19 spread, arXiv preprint arXiv:2004.04565

72. Velásquez et al. N (2020) Hate multiverse spreads malicious COVID-19 content online beyond individual platform control, arXiv preprint arXiv:2004.00673

73. de Montjoye Y-A, Houssiau F (2020) Can we fight COVID-19 without resorting to mass surveillance?, CPG Blog

74. Grimmond T COVID-19: trustworthy facts and waste handling guidelines

75. Marston HR, Musselwhite C, Hadley R COVID-19 vs social isolation: the impact technology can have on communities. Soc Connect Citiz

76. Greenhalgh T, Wherton J, Shaw S, Morrison C (2020) Video consultations for covid-19, ed: British Medical Journal Publishing Group

77. Cho H, Ippolito D, Yu YW (2020) Contact tracing mobile apps for COVID-19: privacy considerations and related trade-offs, arXiv preprint arXiv:2003.11511

78. Aldwairi M, Hasan M, Balbahaith Z Detection of drive-by download attacks using machine learning approach, in Cognitive Analytics: Concepts, Methodologies, Tools, and Applications: IGI Global, 2020, pp. 1598-1611

79. Arabi YM, Murthy S, Webb S (2020) COVID-19: a novel coronavirus and a novel challenge for critical care. Intensive Care Med 46(5):833-836

80. Willan J, King AJ, Jeffery K, Bienz N (2020) Challenges for NHS hospitals during covid-19 epidemic, ed: British Medical Journal Publishing Group

81. Chatter MTJI, Home Literacy Learning During COVID-19

82. Baker SR, Farrokhnia R, Meyer S, Pagel M, Yannelis C, Spending Respond How Does Household, to an Epidemic? Consumption During the, (2020) COVID-19 pandemic. Natl Bur Econ Res 08982937:2020

83. Singh R, Adhikari R (2020) Age-structured impact of social distancing on the COVID-19 epidemic in India, arXiv preprint arXiv:2003.12055

Publisher's Note Springer Nature remains neutral with regard to jurisdictional claims in published maps and institutional affiliations. 


\section{Affiliations}

\section{Suleman Khan ${ }^{1} \cdot$ Muhammad Khurram Khan ${ }^{2}$ (D) Rizwan Khan ${ }^{3}$}

$凶 \quad$ Muhammad Khurram Khan

mkhurram@ksu.edu.sa

Suleman Khan

suleman.khan@northumbria.ac.uk

Rizwan Khan

im.rk86khan@gmail.com

1 Department of Computer and Information Sciences, Northumbria University, Newcastle, Upon Tyne, NE1 8ST, United Kingdom

2 College of Computer \& Information Sciences, King Saud University, Riyadh 11653, Saudi Arabia

3 Institute of Management Sciences (IM-Sciences), Peshawar, Pakistan 